\title{
Nilpotent dynamics on signed interaction graphs and weak converses of Thomas' rules
}

\author{
Adrien Richard *†£
}

January 22, 2018; revised March 282019

\begin{abstract}
A finite dynamical system with $n$ components is a function $f: X \rightarrow X$ where $X=$ $X_{1} \times \cdots \times X_{n}$ is a product of $n$ finite intervals of integers. The structure of such a system $f$ is represented by a signed $\operatorname{digraph} G$, called interaction graph: there are $n$ vertices, one per component, and the signed arcs describe the positive and negative influences between them. Finite dynamical systems are usual models for gene networks. In this context, it is often assumed that $f$ is degree-bounded, that is, the size of each $X_{i}$ is at most the out-degree of $i$ in $G$ plus one. Assuming that $G$ is connected and that $f$ is degree-bounded, we prove the following: if $G$ is not a cycle, then $f^{n+1}$ may be a constant. In that case, $f$ describes a very simple dynamics: a global convergence toward a unique fixed point in $n+1$ iterations. This shows that, in the degree-bounded case, the fact that $f$ describes a complex dynamics cannot be deduced from its interaction graph. We then widely generalize the above result, obtaining, as immediate consequences, other limits on what can be deduced from the interaction graph only, as the following weak converses of Thomas' rules: if $G$ is connected and has a positive (negative) cycle, then $f$ may have two (no) fixed points.
\end{abstract}

Keywords: Finite dynamical system, interaction graph, feedback cycle, fixed point, gene network.

\section{Introduction}

Let $X=X_{1} \times \cdots \times X_{n}$ be a product of $n$ finite intervals of integers, and let $f: X \rightarrow X$,

$$
x=\left(x_{1}, \ldots, x_{n}\right) \mapsto f(x)=\left(f_{1}(x), \ldots, f_{n}(x)\right) .
$$

Such a function $f$ is regarded as a finite dynamical system with $n$ components (FDS for short). If $X=\{0,1\}^{n}$ then $f$ is usually called a Boolean network (BN for short). FDSs have many applications. In particular, since the seminal works of MacCulloch and Pitts, Hopfield, Kauffman and Thomas, they are classical models for the dynamics of neural and gene networks [16, 10, 8, 11, 12, 31, 33. They are also used in information theory, for the network coding problem [6, 5].

The structure of a FDS $f$ is usually represented by a signed digraph, called interaction graph. Formally, a signed digraph is a couple $G=(V, E)$ where $V$ is a set of vertices and $E \subseteq V \times V \times\{+,-\}$ is a set of signed arcs: if $(j, i, s) \in E$ then $G$ has an arc from $j$ to $i$ of sign $s$. The interaction graph of $\boldsymbol{f}$ is then defined as the signed digraph with vertex set $\{1, \ldots, n\}$ and with a positive (resp. negative) arc from $j$ to $i$ if there exists $x \in X$ with $x_{j}<\max \left(X_{j}\right)$ such that

$$
f_{i}\left(x_{1}, \ldots, x_{j}+1, \ldots, x_{n}\right)-f_{i}\left(x_{1}, \ldots, x_{j}, \ldots, x_{n}\right)
$$

\footnotetext{
${ }^{*}$ Laboratoire I3S, UMR CNRS7271, Université Côte d'Azur, France

${ }^{\dagger}$ CMM,UMI CNRS 2807, Universidad de Chile, Chile

${ }^{\ddagger}$ This work is partially supported by the Young Researcher project ANR-18-CE40-0002-01 "FANs", and project STIC AmSud CoDANet 19-STIC-03 (Campus France 43478PD).

${ }^{\S}$ E-mail adress: richard@unice.fr
} 
is positive (resp. negative). If $G$ is the interaction graph of $f$, we say that $f$ is a FDS on $\boldsymbol{G}$.

In molecular biology, the first reliable informations available about a biological system often concern its interaction graph [35, 15]. A natural question is then the following: what can be said on the dynamics of $f$ according to its interaction graph only? Among the many dynamical properties that can be studied, fixed points are of special interest, since they correspond to stable states and often have a strong meaning [25, 33, 8]. For instance, in the context of gene networks, they correspond to stable patterns of gene expression at the basis of particular biological processes 33 . As such, they are the property which has been studied the most.

In particular, many works have been stimulated by two general rules, stated in 1981 by the biologist René Thomas [32, concerning the influence of positive and negative cycles of the interaction graph (cycles are always directed and without repeated vertices, and the sign of a cycle is the product of the signs of its arcs). Roughly, the first (resp. second) rule says that the presence of a positive (resp. negative) cycle is a necessary condition for multiple stable states (resp. permanent periodic behaviors). Several variations of these rules have been formally stated and proved for several classes of discrete and continuous dynamical systems (see e.g. [2, 18, 24, 19, 20, 22, 23] for the discrete case, and [17, 27, 9, 4, 29, 30, 14, 28, 13, for the continuous case). In particular, the first rule has many generalizations and strong versions involving local interaction graphs based on the Jacobian matrix (see [23, 13] and the references therein). It has also a nice biological interpretation since multiple stable states can account for cell differentiation processes [34].

In this paper, we are mainly concerned with the basic discrete versions of Thomas' rules given by the second and third item of the following theorem. From them we deduce that there is a unique fixed point in the acyclic case, and the first item says something stronger $\left(f^{k}\right.$ denotes the $k$ th composition of $f$ with itself).

Theorem 1. Let $f$ a FDS with $n$ components on a signed digraph $G$.

(a) If $G$ has no cycle, then $f^{n}$ is constant, and thus $f$ has a unique fixed point [25, 26].

(b) If $G$ has no negative cycle, then $f$ has at least one fixed point [21].

(c) If $G$ has no positive cycle, then $f$ has at most one fixed point [18, 24].

The following complementary theorem shows that, in the the Boolean case, stronger conclusions can be obtained under the additional assumption that the interaction graph is strongly connected (i.e. for every distinct vertices $i$ and $j$, there is a path from $i$ to $j$ ).

Theorem 2. Let $f$ be a BN on a strongly connected signed digraph $G$ with at least one arc.

(a) If $G$ has no negative cycle, then $f$ has at least two fixed points [2].

(b) If $G$ has no positive cycle, then $f$ has no fixed point [2].

The above results give conditions on $G$ that force all the FDSs or BNs on $G$ to have particular properties. In this paper, we take the converse direction: we study conditions on $G$ that allow at least one FDS on $G$ to have particular properties. Since it is clearly easier to exhibit a particular FDS when we allow the intervals $X_{i}$ to be large, it is natural to think to bound the size of these intervals. This leads us to focus on degree-bounded systems, which are usual in the context of gene networks. The formal definition follows.

Let $f$ be an FDS with $n$ components, and let $G$ be its interaction graph. We say that $f$ is degree-bounded if, for all $i \in\{1, \ldots, n\}$,

- $\left|X_{i}\right|=2$ if $d_{G}^{\text {out }}(i)=0<d_{G}^{\text {in }}(i)$, and

- $\left|X_{i}\right| \leq d_{G}^{\text {out }}(i)+1$ otherwise. 
Here, $d_{G}^{\text {out }}(i)$ is the out-degree of $i$ in $G$, defined as the number of positive arcs leaving $i$ plus the number of negative $\operatorname{arcs}$ leaving $i$. The in-degree $d_{G}^{\text {in }}(i)$ is defined similarly.

These conditions on the intervals $X_{i}$ could seem unnatural at first glance. However, René Thomas, who introduced them, showed that they are very natural in the context of gene networks. He actually widely popularized the use of degree-bounded systems for modeling gene networks through the so called generalized logical method; see [33, 35] for a presentation, and see [1] and the references therein for recent applications. The intuition behind the degree-bounded conditions is the following. In continuous modelings of gene networks, the influence of an interaction from $i$ to $j$ of sign $s$ is often described by a sigmoid function, which can be approximated by a step function with a threshold $\theta_{i j}^{s}$. From this approximation, the system can be discretized in a natural way: the variable $x_{i}$, which is a non negative real number representing a concentration, is approximated by a discrete variable $\tilde{x}_{i}$ defined as the number of out-going interactions $(i, j, s)$ such that $x_{i} \geq \theta_{i j}^{s}$. The size of the domain of this discrete variable $\tilde{x}_{i}$ is thus at most the number of interactions leaving $i$ plus one (see [33, 35] for details).

\section{$1.1 \quad$ Nilpotent systems}

Our first result concerns nilpotent FDSs, that is, FDSs $f$ such that $f^{k}$ is constant for some positive integer $k$.

Theorem 3. If $G$ is an n-vertex connected signed digraph distinct from a signed cycle, then there exists a degree-bounded system $f$ on $G$ such that $f^{n+1}$ is constant.

This shows that a nilpotent degree-bounded system with a linear convergence can be defined on almost all connected signed digraphs. Since such a nilpotent system describes a very simple dynamics - a global convergence in $n+1$ iterations - this shows that the fact that a degreebounded system describes a complex dynamics cannot be deduced from its interaction graph.

If $G$ is a signed cycle, then any degree-bounded system $f$ on $G$ is a $\mathrm{BN}$, and we deduce from Theorem 2 that $f$ has either zero or two fixed points, thus $f$ is not nilpotent. Therefore, according to the previous theorem, we have the following characterization:

Corollary 1. Let $G$ be a connected signed digraph. There exists a nilpotent degree-bounded system on $G$ if and only if $G$ is not a signed cycle.

The existence of nilpotent systems is also the subject of the recent paper [7]. It is proved that, for every $n$-vertex signed digraph $G$,

- there exists a FDS $f: X \rightarrow X$ on $G$ with $X=\{0,1,2,3\}^{n}$ such that $f^{2}$ is constant, and

- there exists a FDS $f: X \rightarrow X$ on $G$ with $X=\{0,1,2\}^{n}$ such that $f^{\left\lfloor\log _{2} n\right\rfloor+2}$ is constant.

As shown above, there does not necessarily exist a nilpotent $\mathrm{BN}$ on a given signed digraph $G$, and [7] provides several sufficient conditions for the existence of such a BN. This raises the question of a characterization of the signed digraphs on which a nilpotent BN can be defined, in the spirit of the previous corollary, which gives such a characterization in the degree-bounded case. In [7] the following question is also raised.

Question 1. Is there a constant c such that, for every n-vertex signed digraph $G$, if there exists a nilpotent $B N$ on $G$, then there exists a nilpotent $B N f$ on $G$ that converges in at most cn iterations, that is, such that $f^{c n}$ is constant?

Theorem 3 and Corollary 1 clearly give a positive answer in the degree-bounded case. 


\subsection{Convergence between systems and weak converses of Thomas' rules}

Our second result generalizes the previous one using the following notion of convergence between systems. Let $f: X \rightarrow X$ and $h: Y \rightarrow Y$ be two FDSs with $n$ components. We say that $f$ converges toward $\boldsymbol{h}$ in $k$ steps if

- $f^{k}(X) \subseteq h(Y) \subseteq Y \subseteq X$, and

- $f(x)=h(x)$ for all $x \in Y$.

In a signed digraph, a source is a vertex of in-degree zero, a sink is a vertex of out-degree zero, and an isolated vertex is a vertex of in- and out-degree zero.

Theorem 4. Let $G$ be a connected signed digraph distinct from a signed cycle. Let $H$ be a subgraph of $G$ obtained by removing arcs only. Suppose that every non-isolated source of $H$ is a source of $G$, and that every non-isolated sink of $H$ is a sink of $G$. Then, for every degree-bounded system $h$ on $H$, there exists a degree-bounded system $f$ on $G$ that converges toward $h$ in $k+1$ steps, where $k$ is the number of isolated vertices in $H$.

Before discussing some consequences of this theorem, let us first observe that some conditions on the subgraph $H$ are needed to obtain the conclusion. Suppose, for instance, that $H$ is obtained from $G$ by removing an arc from $i$ to $j$ with $d_{G}^{\text {in }}(i)=d_{G}^{\text {out }}(i)=d_{G}^{\text {in }}(j)=1$, and let $h: Y \rightarrow Y$ be any degree-bounded system on $H$. Then there is no degree-bounded system $f: X \rightarrow X$ on $G$ that converges toward $h$. Indeed, suppose, for a contradiction, that such a $f$ exists. Since $d_{H}^{\text {out }}(i)<d_{G}^{\text {out }}(i)=d_{H}^{\text {in }}(i)=1, X_{i}$ and $Y_{i}$ are of size two, and thus $X_{i}=Y_{i}$. Since $f_{j}$ only depends on $x_{i}$ and $X_{i}=Y_{i}$, there exists $x, y \in Y$ that only differ in $x_{i} \neq y_{i}$ such that $f_{j}(x) \neq f_{j}(y)$. Since $f$ converges toward $h$, we deduce that $h_{j}(x) \neq h_{j}(y)$ and thus $d_{H}^{\text {in }}(j)>0$, a contradiction.

A first immediate consequence of Theorem 4 is Theorem 3. Indeed, consider the subgraph $H$ obtained by removing all the arcs of $G$, and let $h: Y \rightarrow Y$ be any degree-bounded system on $H$. Then $H$ consists in $n$ isolated vertices, and thus $Y$ is a singleton, so that $h:\{\xi\} \rightarrow\{\xi\}$ for some $\xi \in \mathbb{Z}^{n}$. Then, according to the conclusion, there exists a degree-bounded system $f$ on $G$ that converges toward $h$ in $n+1$ steps, thus $f^{n+1}=\mathrm{cst}=\xi$, and Theorem 3 is recovered.

As another consequence, we have the following weak converses of Theorems 1(b) and 1(c).

Corollary 2. Let $G$ be a connected signed digraph.

(a) If $G$ has a negative cycle, then there exists a degree-bounded system on $G$ without fixed point.

(b) If $G$ has a positive cycle, then there exists a degree-bounded system on $G$ with two fixed points.

(c) More generally, if $G$ has $k$ vertex-disjoint positive cycles, with $k \geq 1$, then there exists a degree-bounded system on $G$ with $2^{k}$ fixed points.

Proof. For the first point, suppose that $G$ has a negative cycle $C$, and let $H$ be obtained from $G$ by removing all the arcs of $G$, excepted those of $C$. Let $h$ be any degree-bounded system on $H$. It is easy to see that $h$ has no fixed point. If $G=C$ then $G=H$ and we are done. Otherwise, by Theorem 4 , there exists a degree-bounded system $f$ on $G$ that converges toward $h$. Thus $f$ has no fixed point.

The proof of the third point, which implies the second, is similar. Suppose that $G$ has $k$ vertex-disjoint positive cycles, say $C_{1}, \ldots, C_{k}$. Let $H$ be obtained from $G$ by removing all the arcs of $G$, excepted those of $C_{i}, 1 \leq i \leq k$. Let $h$ be any degree-bounded system on $H$. It is easy to see that $h$ has $2^{k}$ fixed points. If $k=1$ and $G=C_{1}$, then $G=H$ and we are done. Otherwise, by Theorem 4 , there exists a degree-bounded system $f$ on $G$ that converges toward $h$. Thus $f$ has $2^{k}$ fixed points. 
Note that from Thomas' rules and the above weak converses, we obtain the following characterizations: if $G$ is connected, then $G$ has a negative (positive) cycle if and only if there exists a degree-bounded system on $G$ no (two) fixed points.

Note also that the weak converses of Thomas' rules are false in the Boolean case. For instance, if $G$ consists in two cycles with opposite signs that share exactly one vertex, then it is easy to see that every $\mathrm{BN}$ on $G$ has a unique fixed point. More generally, if $G$ is strongly connected, has a unique negative (positive) cycle, and has at least one positive (negative) cycle, then every BN on $G$ has at least (at most) one fixed point [22].

Another gap between the Boolean case and the degree-bounded case, that shows the relevance of Corollary 2(c), is the following. Let $\nu^{+}$be the maximum number of vertex-disjoint positive cycles in $G$. According to Corollary 2(c), the maximum number of fixed points in a degreebounded system on $G$ is exponential in $\nu^{+}$while, in the Boolean case, it can be linear in $\nu^{+}$. Indeed, as proved in [3], for every positive integer $k$ there exists a strongly connected signed digraph $G_{k}$ with $k$ vertex-disjoint positive cycles such that every BN on $G_{k}$ has at most $k+1$ fixed points.

The organization of the paper is the following. A slightly stronger version of Theorem 3 is proved in Section 2, and the proof of Theorem 4, which uses this stronger version, is given in Section 3 ,

\section{Nilpotent systems}

Let $G=(V, E)$ be a signed digraph. The set of positive in-neighbors of $i$ in $G$, denoted $G_{i}^{+}$, is the set of $j \in V$ such that $(j, i,+) \in E$. The set of negative in-neighbors is defined similarly. The set of in-neighbors of $i$ in $G$ is $G_{i}=G_{i}^{+} \cup G_{i}^{-}$. These notations are rather unconventional, but useful in the following for their compactness. Note that $G_{i}^{+}$and $G_{i}^{-}$are not necessarily disjoint: if $j \in G_{i}^{+} \cap G_{i}^{-}$then $G$ has both a positive and a negative arc from $j$ to $i$, and we say that $G$ has parallel arcs from $j$ to $i$. Note also that $d_{G}^{\text {in }}(i)=\left|G_{i}^{+}\right|+\left|G_{i}^{-}\right|$(thus the in-degree can be greater than the number of in-neighbors and, similarly, the out-degree may be greater than the number of out-neighbors). We denote by $|G|$ be the underlying unsigned digraph of $G$ : the vertex set is $V$ and there is an arc from $j$ to $i$ if and only if $j \in G_{i}$. See Figure 1 for an illustration. We say that $G$ is a signed cycle if $|G|$ is a cycle and $G$ has no parallel arc (thus the signed digraph in Figure 1 is not a signed cycle, while its underlying unsigned digraph is a cycle). If $G^{\prime}=\left(V^{\prime}, E^{\prime}\right)$ is another signed digraph, then $G \cup G^{\prime}=\left(V \cup V^{\prime}, E \cup E^{\prime}\right)$. The subgraph of $G$ induced by $U \subseteq V$, denoted $G[U]$, is the signed digraph with vertex set $U$ and arc set $E \cap U \times U \times\{+,-\}$. We set $G \backslash U=G[V \backslash U]$. We say that $U$ is a strong component of $G$ is $G[U]$ is strongly connected and $U$ is maximal for this property. A strong component $U$ is initial if $G$ has no arc from $V \backslash U$ to $U$. A strong component $U$ is trivial if $G[U]$ is the trivial graph, i.e. has one vertex and no arc.

We say that $G$ is basic is all the initial strong components of $G$ are trivial. We set

$$
\beta(G)= \begin{cases}0 & \text { if } G \text { is basic } \\ 1 & \text { otherwise }\end{cases}
$$

If $i, j \in V$ then $d_{G}(j, i)$ denotes the minimum number of arcs in a path from $j$ to $i$. If $j=i$ then $d_{G}(j, i)=0$ and if there is no path from $j$ to $i$ then $d_{G}(j, i)=\infty$. If $I \subseteq V$ then $d(I, i)$ is the minimum of $d(j, i)$ for $j \in I$. Let $\mathcal{I}$ be the set of initial strong components of $G$. We set

$$
\lambda(G)=\max _{i \in V} \min _{I \in \mathcal{I}} d_{G}(I, i)+|I| .
$$

Remark 1. Suppose that $G$ has $n$ vertices. Clearly, for every vertex $i$, there exists $I^{*} \in \mathcal{I}$ such that $G$ has a path from $I^{*}$ to $i$. If $P$ is a shortest path from $I^{*}$ to $i$, then, excepted the initial 


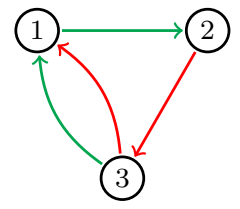

$G$

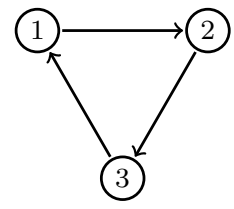

$|G|$

Figure 1: Positive (negative) arcs are green (red). This convention is used throughout the paper.

vertex of $P$, no vertex belong to an initial strong component of $G$. We deduce that the number of $\operatorname{arcs}$ in $P$ is at most $n-\sum_{I \in \mathcal{I}}|I|$. As a consequence,

$$
\lambda(G) \leq n-\sum_{I \in \mathcal{I}}|I|+\max _{I \in \mathcal{I}}|I| \leq n .
$$

In particular, if $G$ has at least two initial strong components then $\lambda(G)<n$, and if $G$ is basic and has $k$ sources, then $\lambda(G) \leq n-k+1$. Note also that $\lambda(G)=n$ if $G$ is strongly connected.

We are now in position to state the following quantitative version of Theorem 3 . We write $f^{k}=\mathrm{cst}=\xi$ to mean that $f^{k}$ is a constant function, always equal to $\xi$.

Theorem 5. Let $G$ be a connected signed digraph distinct from a signed cycle. There exists a degree-bounded system $f: X \rightarrow X$ on $G$ such that

$$
f^{\lambda(G)+\beta(G)}=\mathrm{cst}=\xi
$$

where $\xi_{i}=\min \left(X_{i}\right)$ for all sources $i$ of $G$.

Proof. Suppose that $G$ has vertex set $V=\{1, \ldots, n\}$. If $G$ is trivial the theorem is obvious, so assume that $G$ is not trivial. The proof is devised in two cases.

Case 1: $|\boldsymbol{G}|$ is not a cycle. Let $\mathcal{I}=\left\{I_{1}, \ldots, I_{k}\right\}$ be the set of initial strong components of $G$. Since $G$ is connected and since $|G|$ is not a cycle, each $I_{\ell}$ contains at least one vertex $i_{\ell}$ such that either $i_{\ell}$ is a source or, for all $j \in G_{i_{\ell}}$, the out-degree of $j$ in $|G|$ is at least two. Let $H$ be the subgraph obtained from $G$ by removing all $\operatorname{arcs}$ with $i_{\ell}$ as terminal vertex, $\ell=1, \ldots, k$. The initial strong components of $H$ are $\left\{i_{1}\right\}, \ldots,\left\{i_{k}\right\}$ thus $H$ is basic. Furthermore, for every $\ell=1, \ldots, k$ and $j \in G_{i_{\ell}}$, the out degree of $j$ in $H$ is at least one. Hence,

$$
\text { every sink of } H \text { is a sink of } G \text {. }
$$

Furthermore,

$$
\lambda(H) \leq \lambda(G) .
$$

Indeed, let $i \in V$ and let any $\ell$ such that $G$ has a path $P$ from $I_{\ell}$ to $i$. Suppose that $P$ is of length $d_{G}\left(I_{\ell}, i\right)$. Let $j$ be the first vertex of $P$, and let $P^{\prime}$ be a path from $i_{\ell}$ to $j$ of length $d_{G}\left(i_{\ell}, j\right)$. Since $P^{\prime} \cup P$ is a path of $H, d_{H}\left(i_{\ell}, i\right) \leq d_{G}\left(i_{\ell}, j\right)+d_{G}\left(I_{\ell}, i\right)$, and since $P^{\prime}$ is contained in $G\left[I_{\ell}\right]$ we have $d_{G}\left(i_{\ell}, j\right)<\left|I_{\ell}\right|$. Hence $d_{H}\left(i_{\ell}, i\right)+1 \leq d_{G}\left(I_{\ell}, i\right)+\left|I_{\ell}\right|$. It follows that $\lambda(H) \leq \lambda(G)$.

Let $I=\left\{i_{1}, \ldots, i_{k}\right\}$ and let $X=X_{1} \times \cdots \times X_{n}$ be defined as follows: for all $i \in V$,

$$
X_{i}= \begin{cases}\{0,1,2,3\} & \text { if } i \in G_{j}^{+} \cap G_{j}^{-} \text {for some } j \in I \\ \{0,1,2\} & \text { if } i \in G_{j}^{+} \backslash G_{j}^{-} \text {for some } j \in I \\ \{0,1,2\} & \text { if } i \in G_{j}^{-} \backslash G_{j}^{+} \text {for some } j \in I \\ \{0,1,2\} & \text { if } i \in G_{j}^{+} \cap G_{j}^{-} \text {for some } j \in V \backslash I \\ \{0,1\} & \text { otherwise. }\end{cases}
$$

Let $f: X \rightarrow X$ be any FDS on $G$. Since $G$ has no isolated vertex, to prove that $f$ is degreebounded, it is sufficient to prove that $\left|X_{i}\right| \leq \max \left(2, d_{G}^{\text {out }}(i)+1\right)$ for every $i$. This is obvious in 
cases (4) and (5). In case (1) we have $d_{G}^{\text {out }}(i) \geq 2$, and if $d_{G}^{\text {out }}(i)=2$, then $i$ is a sink of $H$ but not a sink of $G$, a contradiction with (荬). Hence $d_{G}^{\text {out }}(i) \geq 3$ and thus $\left|X_{i}\right|=4 \leq d_{G}^{\text {out }}(i)+1$. Similarly, in case (2) and (3) we have $d_{G}^{\text {out }}(i) \geq 1$, and if $d_{G}^{\text {out }}(i)=1$, then $i$ is a sink of $H$ but not a sink of $G$, a contradiction with (図). Hence $d_{G}^{\text {out }}(i) \geq 2$ and thus $\left|X_{i}\right|=3 \leq d_{G}^{\text {out }}(i)+1$. Thus every FDS $f: X \rightarrow X$ on $G$ is degree-bounded.

For $1 \leq p \leq \lambda(H)$, let $J_{p}$ be the set of $i \in V$ such that $d_{H}(I, i)+1=p$. In this way, $J_{1}=I$, and for all $i \in J_{p}$ with $p \geq 2, G$ has at least on arc from $J_{p-1}$ to $i$. Furthermore, since $\lambda(H)=\max _{i \in V} d_{H}(I, i)+1$, every vertex $i \in V$ is contained in some $J_{p}$.

Let $\xi \in X$ be inductively defined as follows:

- For all $i \in J_{1}$ :

$$
\xi_{i}= \begin{cases}1 & \text { if } G_{i}^{-} \backslash G_{i}^{+} \neq \emptyset \\ 0 & \text { otherwise }\end{cases}
$$

- For all $i \in J_{p}$ with $p \geq 2$ :

$$
\xi_{i}= \begin{cases}1 & \text { if } \xi_{j}=1 \text { for all } j \in G_{i}^{+} \cap J_{p-1} \\ & \text { and } \xi_{j}=0 \text { for all } j \in\left(G_{i}^{-} \backslash G_{i}^{+}\right) \cap J_{p-1} \\ 0 & \text { otherwise. }\end{cases}
$$

Finally, let $f: X \rightarrow X$ be defined as follows:

- For all $i \in J_{1}$ :

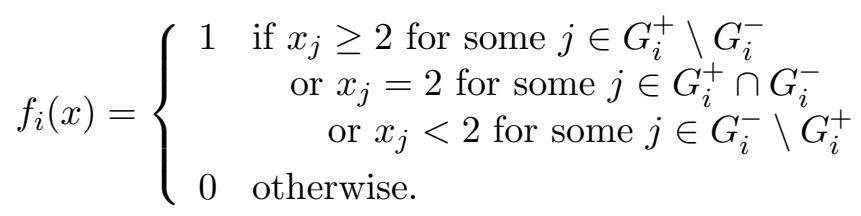

- For all $i \in V \backslash J_{1}$ with $\xi_{i}=1$ :

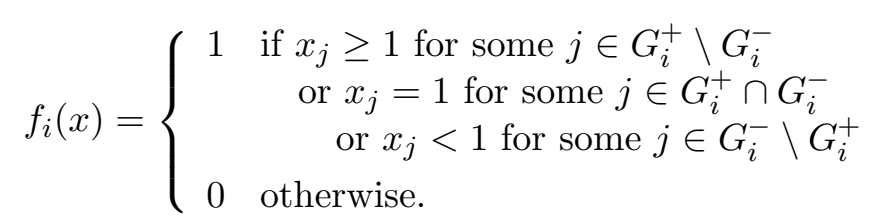

- For all $i \in V \backslash J_{1}$ with $\xi_{i}=0$ :

$$
f_{i}(x)=\left\{\begin{array}{cc}
1 \quad & \text { if } x_{j} \geq 1 \text { for all } j \in G_{i}^{+} \backslash G_{i}^{-} \\
& \text {and } x_{j}=1 \text { for all } j \in G_{i}^{+} \cap G_{i}^{-} \\
& \quad \text { and } x_{j}<1 \text { for all } j \in G_{i}^{-} \backslash G_{i}^{+} \\
0 & \text { otherwise. }
\end{array}\right.
$$

It is straightforward to prove that $G$ is the interaction graph of $f$. Let $\beta=\beta(G)$. Since $\lambda(H) \leq \lambda(G)$, and according to the definition of $\xi$, to conclude the proof it is sufficient to prove that $f^{\lambda(H)+\beta}=\mathrm{cst}=\xi$. For that we prove the following:

$$
\forall 1 \leq p \leq \lambda(H), \forall i \in J_{p}, \forall \ell \geq p, \quad f_{i}^{\ell+\beta}=\mathrm{cst}=\xi_{i}
$$

We proceed by induction on $p$.

- Let $i \in J_{1}$. If $\beta=0$ then $i$ is a source of $G$, thus $G_{i}^{-} \backslash G_{i}^{+}=\emptyset$ and we deduce that $f_{i}=\operatorname{cst}=0=\xi_{i}$, thus $f_{i}^{\ell}=\operatorname{cst}=\xi_{i}$ for all $\ell \geq 1$. Suppose now that $\beta=1$. Let $x \in X$ and $\ell \geq 1$. Since $f(X) \subseteq\{0,1\}^{n}$ we have $f^{\ell}(x) \in\{0,1\}^{n}$. If $\xi_{i}=1$ there exists $j \in G_{i}^{-} \backslash G_{i}^{+}$ and since $f_{j}^{\ell}(x)<2$ we have $f_{i}\left(f^{\ell}(x)\right)=1=\xi_{i}$. If $\xi_{i}=0$ then $G_{i}^{-} \backslash G_{i}^{+}=\emptyset$, and since $f_{j}^{\ell}(x)<2$ for all $j \in G_{i}^{+}$, we deduce that $f_{i}\left(f^{\ell}(x)\right)=0=\xi_{i}$. Thus $f_{i}^{\ell+1}=$ cst $=\xi_{i}$ in all cases. 
- Suppose that $i \in J_{p}$ with $p \geq 2$. Let $x \in X$ and $\ell \geq p$. If $\xi_{i}=1$ then by the definition of $\xi$ and the induction hypothesis, we have $f_{j}^{\ell-1+\beta}(x)=\xi_{j}=1$ for all $j \in G_{i}^{+} \cap J_{p-1}$ and $f_{j}^{\ell-1+\beta}(x)=\xi_{j}=0$ for all $j \in\left(G_{i}^{-} \backslash G_{i}^{+}\right) \cap J_{p-1}$. Since $\left(G_{i}^{+} \cup G_{i}^{-}\right) \cap J_{p-1}$ is not empty, we deduce from the definition of $f_{i}$ that $f_{i}\left(f^{\ell-1+\beta}(x)\right)=1=\xi_{i}$. If $\xi_{i}=0$ then by the definition of $\xi$ and the induction hypothesis, we have $f_{j}^{\ell^{\prime}}(x)=\xi_{j}=0$ for some $j \in G_{i}^{+} \cap J_{p-1}$ or $f_{j}^{\ell^{\prime}}(x)=\xi_{j}=1$ for some $j \in\left(G_{i}^{+} \backslash G_{i}^{-}\right) \cap J_{p-1}$, and we deduce from the definition of $f_{i}$ that $f_{i}\left(f^{\ell-1+\beta}(x)\right)=0=\xi_{i}$. Thus $f_{i}^{\ell+\beta}=$ cst $=\xi_{i}$ in all cases.

This proves (隹) and completes the proof of the first case. See Example 1 for an illustration.

Case 2: $|\boldsymbol{G}|$ is a cycle. Then $\lambda(G)=n$ and $\beta(G)=1$, and since $G$ has no source, we have to prove that there exists a degree-bounded $f$ system on $G$ such that $f^{n+1}=$ cst. Without loss of generality, suppose that $|G|$ has an arc from $i$ to $i+1$ for all $1 \leq i<n$ and an arc from $n$ to 1. For convenience, identify $n+1$ with 1 and 0 with $n$. Let $I$ be the set of vertices $i$ such that $G$ has parallel $\operatorname{arcs}$ from $i$ to $i+1$. Since $G$ is not a signed cycle and since $|G|$ is a cycle (as in Figure 11), $I$ is not empty, and without loss of generality we assume that $n \in I$.

Let $X=X_{1} \times \cdots \times X_{n}$ be defined by

$$
X_{i}= \begin{cases}\{0,1,2\} & \text { if } i \in I \\ \{0,1\} & \text { otherwise. }\end{cases}
$$

Let $f: X \rightarrow X$ be defined by

$$
f_{i}(x)= \begin{cases}\max \left(X_{i}\right) \quad & \text { if } x_{i-1}=1 \text { and } i-1 \in G_{i}^{+} \\ & \text {or } x_{i-1}=0 \text { and } i-1 \in G_{i}^{-} \backslash G_{i}^{+} \\ 0 & \text { otherwise. }\end{cases}
$$

It is straightforward to show that $f$ is a degree-bounded system on $G$. Let us prove that $f^{n+1}=$ cst. Since $f_{i}$ only depends on $x_{i-1}$, we can abusively write $f_{i}(x)=f_{i}\left(x_{i-1}\right)$; this allows us to define $\xi \in X$ by induction in the following way: $\xi_{1}=0$ and $\xi_{i}=f_{i}\left(\xi_{i-1}\right)$ for all $2 \leq i \leq n$. Let us prove, by induction on $i$, that $f_{i}^{\ell+1}(x)=\xi_{i}$ for $x \in X$ and $\ell \geq i$. Let $x \in X$. Since $n \in I$, we have $n \in G_{1}^{+} \cap G_{1}^{-}$and $f_{n}^{\ell}(x) \in\{0,2\}$ for all $\ell \geq 1$, thus $f_{1}\left(f^{\ell}(x)\right)=0=\xi_{1}$ and the base case is proved. Let $1<i \leq n$ and $\ell \geq i$. By induction hypothesis $f_{i-1}^{\ell}(x)=\xi_{i-1}$ thus $f_{i}\left(f^{\ell}(x)\right)=f_{i}\left(\xi_{i-1}\right)=\xi_{i}$ and the induction step is proved. Thus $f^{n+1}=$ cst $=\xi$.

Remark 2. The bound $\lambda(G)+\beta(G)$ can be reached. For instance, if $G$ satisfies one of the following conditions, then it is straightforward to prove that there is no degree-bounded system $f$ on $G$ such that $f^{\lambda(G)+\beta(G)-1}$ is constant:

- $G$ is acyclic,

- $G$ is obtained from an acyclic signed digraph by adding a loop on each source,

- $G$ consists of two cycles that intersect in a path.

It could be interesting to characterize the signed digraphs $G$ for which the bound is reached.

Remark 3. Let $G$ be a signed digraph, and let $G_{1}, \ldots, G_{k}$ be its connected components. It is easy to see that $\lambda(G)=\max _{\ell} \lambda\left(G_{\ell}\right)$, and that $\beta(G)=\max _{\ell} \beta\left(G_{\ell}\right)$. Hence, if $G_{\ell}$ is not a signed cycle for all $\ell$, by applying the previous theorem on each connected component, we deduce that there exists a degree-bounded system $f: X \rightarrow X$ on $G$ such that $f^{\lambda(G)+\beta(G)}=\operatorname{cst}=\xi$. 
Example 1. Let $G$ be as follows:

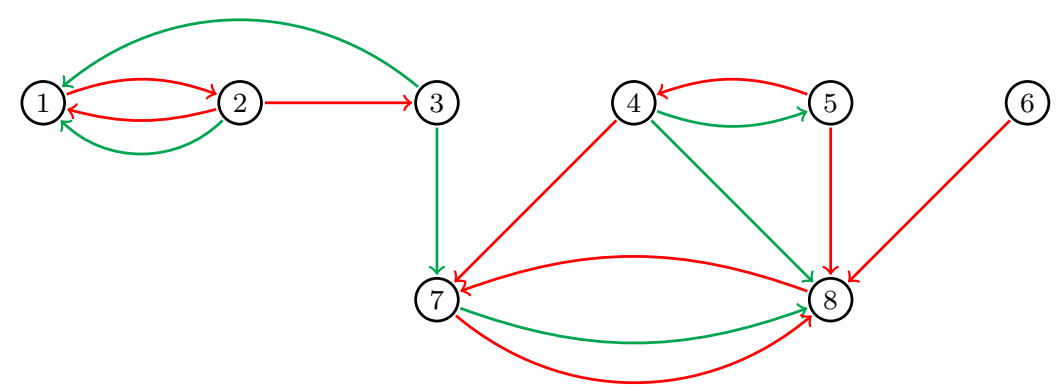

The initial strong components are $I_{1}=\{1,2,3\}, I_{2}=\{4,5\}$ and $I_{3}=\{6\}$. We have $\lambda(G)=3$, and since $I_{1}$ and $I_{2}$ are not trivial, we have $\beta(G)=1$. Then, for the construction described in the proof, we must take $i_{1}=1$ and $i_{3}=6$, while $i_{2}$ should be 4 or 5 . Let us take $i_{2}=4$. Then $I=\{1,4,6\}$ and $X$ is as follows:

$$
\begin{aligned}
& X_{1}=\{0,1\} \\
& X_{2}=\{0,1,2,3\} \\
& X_{3}=\{0,1,2\} \\
& X_{4}=\{0,1\} \\
& X_{5}=\{0,1,2\} \\
& X_{7}=\{0,1,2\} \\
& X_{6}=\{0,1\} \\
& X_{8}=\{0,1\}
\end{aligned}
$$

Besides, $H$ is obtained by removing the parallel arcs from 2 to 1 , the arc from 3 to 1 , and the arc from 5 to 4 . Thus $\lambda(H)=3$ with $J_{1}=I=\{1,4,6\}, J_{2}=\{2,5,7,8\}$, and $J_{3}=\{3\}$. We deduce that

$$
\begin{array}{ll}
\xi_{1}=0 & \xi_{2}=1 \\
\xi_{4}=1 & \xi_{5}=1 \\
\xi_{6}=0 & \xi_{7}=0 \\
\xi_{8}=1
\end{array} \quad \xi_{3}=0 .
$$

and thus $f$ is defined by

$$
\begin{aligned}
& f_{2}(x)=\left\{\begin{array}{l}
1 \text { if } x_{1}<1 \\
0 \text { otherwise }
\end{array}\right. \\
& f_{1}(x)=\left\{\begin{array}{cl}
1 \text { if } x_{2}=2 \\
\text { or } x_{3} \geq 2, \\
0 \text { otherwise }
\end{array}\right. f_{5}(x)=\left\{\begin{array}{l}
1 \text { if } x_{4} \geq 1, \\
0 \text { otherwise }
\end{array}\right. \\
& f_{4}(x)= \begin{cases}1 \text { if } x_{5}<2, \\
0 \text { otherwise }\end{cases} f_{7}(x)=\left\{\begin{array}{c}
1 \text { if } x_{3} \geq 1 \\
\text { and } x_{4}<1 \\
0 \text { otherwise }
\end{array} \quad f_{3}(x)=\left\{\begin{array}{l}
1 \text { if } x_{2}<1, \\
0 \text { otherwise. }
\end{array}\right.\right. \\
& f_{6}(x)=0 \quad \begin{array}{c}
1 \text { if } x_{4} \geq 1 \\
\text { or } x_{5}<1 \\
\text { or } x_{6}<1 \\
\text { or } x_{7}=1
\end{array} \\
& 0 \text { otherwise }
\end{aligned}
$$

It is easy to check that the interaction graph of $f$ is $G$. Furthermore, if $x \in\{0,1\}^{n}$, then it is easy to see that, for all $\ell \geq 1$,

$$
\begin{aligned}
& f_{1}^{\ell}(x)=0=\xi_{1} \quad f_{2}^{\ell+1}(x)=1=\xi_{2} \\
& f_{4}^{\ell}(x)=1=\xi_{2} \quad f_{3}^{\ell+1}(x)=1=\xi_{3} \\
& \begin{array}{ll}
f_{6}^{\ell}(x)=0=\xi_{6} & f_{7}^{\ell+1}(x)=0=\xi_{7} \\
& f_{8}^{\ell+1}(x)=1=\xi_{8}
\end{array} \\
& f_{3}^{\ell+2}(x)=0=\xi_{3} .
\end{aligned}
$$

Since $f(x) \in\{0,1\}^{n}$ for all $x \in X$, we deduce that $f^{4}(x)=\xi$ for all $x \in X$, as desired. 
Example 2. Suppose that $G$ is the 3-vertex signed digraph as in Figure 1;

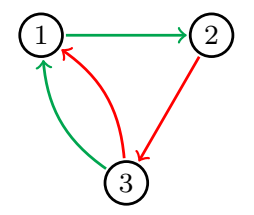

Then $|G|$ is a cycle, and we fall in the second case of the proof. We then have $I=\{3\}$, thus $X_{1}=X_{2}=\{0,1\}$ and $X_{3}=\{0,1,2\}$. Therefore, $f$ is defined by

$$
f_{1}(x)=\left\{\begin{array}{l}
1 \text { if } x_{3}=1 \\
0 \text { otherwise }
\end{array} \quad f_{2}(x)=\left\{\begin{array}{l}
1 \text { if } x_{1}=1 \\
0 \text { otherwise }
\end{array} \quad f_{3}(x)=\left\{\begin{array}{l}
2 \text { if } x_{2}=0 \\
0 \text { otherwise }
\end{array}\right.\right.\right.
$$

It is easy to check that the interaction graph of $f$ is $G$. Furthermore, $f_{3}(x) \neq 1$ for all $x \in X$. Thus, for all $\ell \geq 2$, we have $f_{1}^{\ell}(x)=0$ which implies $f_{2}^{\ell+1}(x)=0$, which implies $f_{3}^{\ell+2}(x)=2$. Thus $f^{4}(x)=(0,0,2)$ for all $x \in X$, as desired.

Example 3. Suppose that $G$ is the 1-vertex signed digraph as follows:

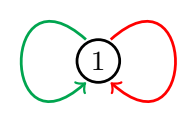

Then $|G|$ is a cycle, and we fall in the second case of the proof. We then have $I=\{1\}$, thus $X=X_{1}=\{0,1,2\}$, and $f=f_{1}$ is defined by $f(0)=0, f(1)=2$ and $f(2)=0$. Thus $f^{2}(x)=0$ for all $x \in X$, as desired.

\section{Convergence between systems}

In this section we prove the following strengthening of Theorem 4, which is more suited for a proof by induction. A spanning subgraph is a subgraph obtained by removing arcs only.

Theorem 6. Let $G$ be signed digraph and let $H$ be a spanning subgraph of $G$. Let $I$ be the set of vertices that are isolated in $H$ but not in $G$. Suppose that every source of $H \backslash I$ is a source of $G$, and that every sink of $H \backslash I$ is a sink of $G$. Suppose also that no connected component of $G$ is a signed cycle with only vertices in $I$. Then, for every degree-bounded system $h$ on $H$ there exists a degree-bounded system $f$ on $G$ that converges toward $h$ in at most $|I|+1$ steps.

The proof of Theorem [6 uses Theorem 5 and the following technical lemma.

Lemma 1. Let $G$ be a signed digraph and let $H$ be a spanning subgraph of $G$ such that

- $G$ has no arc from a sink of $H$ to a source of $H$, and

- $G$ has no arc from a vertex of $H$ to an isolated vertex of $H$.

Let $A$ be the set of sources of $H$ that are not sources of $G$, and let $B$ be the set sinks of $H$ that are not sinks of $G$. Let $h: Y \rightarrow Y$ be a degree-bounded system on $H$ and $\xi \in Y$. There exists a degree-bounded system $f: X \rightarrow X$ on $G$ such that

- $f(X) \subseteq Y$,

- $f_{i}(X) \subseteq h_{i}(Y)$ for all $i \notin A$,

- $f(x)=h(x)$ for all $x \in Y$ such that $x_{i}=\xi_{i}$ for all $i \in B$, and 
- $f_{i}(x)=h_{i}(x)$ for all $x \in Y$ and $i$ such that $G_{i} \cap B=\emptyset$.

Poof of Theorem 6, assuming Lemma 1. Without loss of generality, suppose that $V=$ $\{1, \ldots, n\}$ is the vertex set of $G$ and that $I=\{1, \ldots, m\}$ for some $1 \leq m \leq n$. Let $h: Y \rightarrow Y$ be a degree-bounded system on $H$. Since $h$ is degree-bounded, for all $i \in I$ there exists $\xi_{i} \in \mathbb{Z}$ such that $Y_{i}=\left\{\xi_{i}\right\}$.

Suppose first that $I$ is empty. Then $G$ and $H$ have the same isolated vertices and thus the same sources and sinks. Hence, the conditions of Lemma 1 are satisfied, and the sets $A$ and $B$ of the statement are both empty. Thus there exists a degree-bounded system $f$ on $G$ that converges toward $h$ in one step, and thus $f$ has the desired properties. Hence, in the following, we assume that $I$ is not empty.

We say that $I$ has the property $\boldsymbol{P}$ if every connected component $F$ of $G[I]$ satisfies at least one of the following conditions:

- $F$ is not strongly connected.

- $F$ has a leaving arc, that is, $G$ has an arc from a vertex of $F$ to $V \backslash I$.

- $F$ has no entering arc, that is, $G$ has no arc from $V \backslash I$ to a vertex of $F$.

Case 1: $\boldsymbol{I}$ has the property $\boldsymbol{P}$. Let $\tilde{H}$ be the signed digraph obtained from $H$ by adding all the $\operatorname{arcs}(i, j, s)$ of $G[I]$ such that $G$ has no arc from $i$ to $V \backslash I$. Let $Q$ be the subgraph of $\tilde{H}$ induced by $I$ (see Figure 2). Suppose that some connected component of $Q$ is a cycle $C$. Then $G$ has no arc from $C$ to $V \backslash I$, thus $C$ is a connected component of $G[I]$ without leaving arc. Since no connected component of $G$ is a cycle contained in $G[I]$, we deduce that $C$ has at least one entering arc. Thus $C$ is strongly connected, has no leaving arcs and at least one entering arc. This is not possible since $I$ has the property $P$. Thus no connected component of $Q$ is a cycle. Hence, by applying Theorem 5 on each connected component of $Q$, we deduce (cf. Remark 3 ) that there exists a degree-bounded system $g: Z \rightarrow Z$ on $Q$ such that

$$
g^{\lambda(Q)+\beta(Q)}=\operatorname{cst}=\left(\xi_{1}, \ldots, \xi_{m}\right) .
$$

Let $\tilde{Y}=Z_{1} \times \cdots \times Z_{m} \times Y_{m+1} \times \cdots \times Y_{n}$. Since $H$ has no arc from $I$ to $V \backslash I$, if $i \notin I$ then $h_{i}$ only depends on variables $x_{j}$ with $j \notin I$, and we can define $\tilde{h}: \tilde{Y} \rightarrow \tilde{Y}$ as follows, without possible ambiguity:

$$
\tilde{h}_{i}(x)= \begin{cases}h_{i}\left(x_{m+1}, \ldots, x_{n}\right) & \text { if } i \notin I \\ g_{i}\left(x_{1}, \ldots, x_{m}\right) & \text { if } i \in I .\end{cases}
$$

By construction, $\tilde{h}$ is a degree-bounded system on $\tilde{H}$ such that:

(1) $\tilde{h}_{i}(\tilde{Y}) \subseteq h_{i}(Y)$ for all $i \notin I$,

(2) $\tilde{h}_{i}^{\lambda(Q)+\beta(Q)}(\tilde{Y}) \subseteq\left\{\xi_{i}\right\}=Y_{i}=h_{i}(Y)$ for all $i \in I$,

(3) $\tilde{h}(x)=h(x)$ for all $x \in Y$.

Let $\tilde{G}$ be the signed digraph obtained from $\tilde{H}$ by adding all the $\operatorname{arcs}(i, j, s)$ of $G$ with $j \notin I$ (see Figure 21). Let $A$ be the set of sources of $\tilde{H}$ that are not sources of $\tilde{G}$, and let us prove that $A=\emptyset$. Suppose that $j$ is a source of $\tilde{H}$. If $j \in I$ then $j$ is obviously a source of $\tilde{G}$, and if $j \notin I$ then $j$ is a source of $H \backslash I$. Hence, by hypothesis, $j$ is a source of $G$ and thus of $\tilde{G}$ too. Thus, $A=\emptyset$. This implies that $\tilde{G}$ has no arc from a sink of $\tilde{H}$ to a source of $\tilde{H}$. Furthermore, $\tilde{G}$ has no arc from a vertex of $\tilde{H}$ to an isolated vertex $i$ of $\tilde{H}$ since otherwise $i \notin I$, thus $i$ is isolated in $H$ but not in $G$, a contradiction. Thus $\tilde{G}$ and $\tilde{H}$ satisfy the conditions of Lemma 1 , Let $B$ be the set of sinks of $\tilde{H}$ that are not sinks of $\tilde{G}$. If $i$ is a sink of $\tilde{H}$ and $i \notin I$, then $i$ is a sink of $H \backslash I$ and, by hypothesis, $i$ is $\operatorname{sink}$ of $G$ and thus of $\tilde{G}$ too. Thus $B \subseteq I$. Let $i \in I$ and $j \in B$. 
$G$
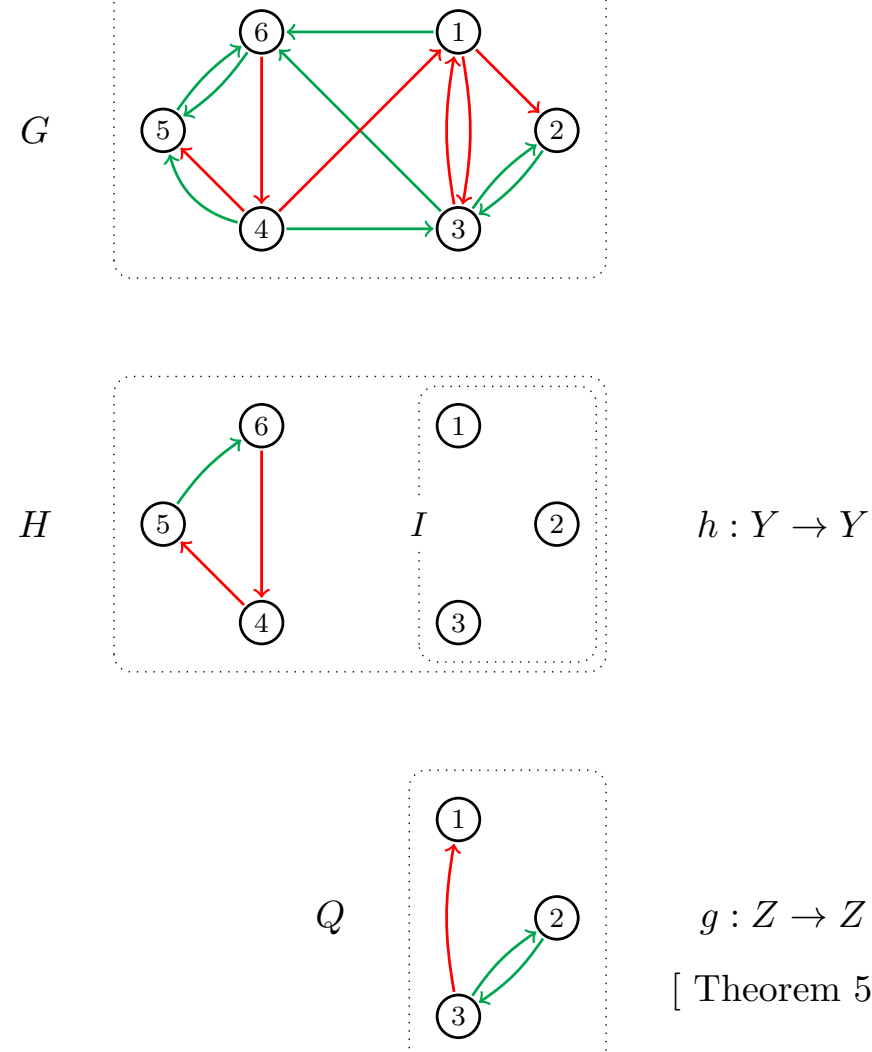

$g: Z \rightarrow Z$

[ Theorem [5]

$\tilde{H}$
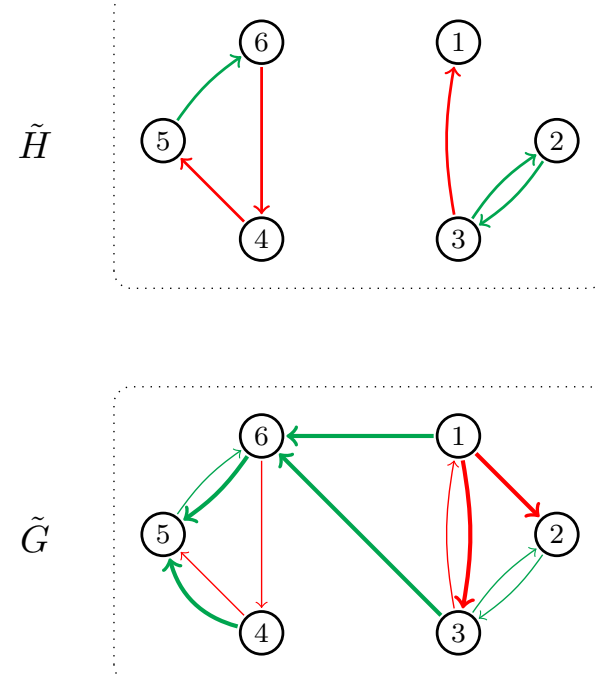

$\tilde{f}: \tilde{X} \rightarrow \tilde{X}$

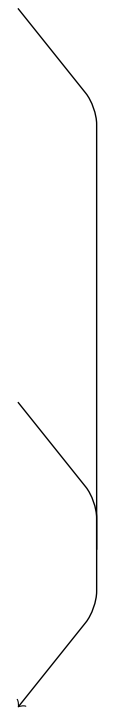

$\tilde{h}: \tilde{Y} \rightarrow \tilde{Y}$

[ Lemma 1]

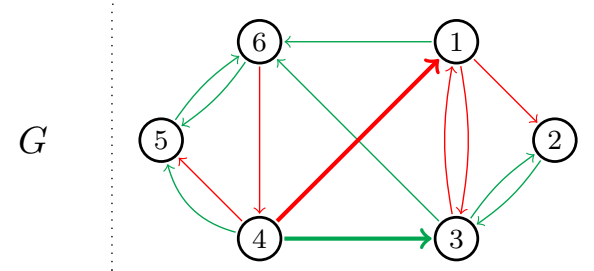

(2) $f: X \rightarrow X$

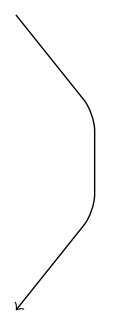

[ Lemma 1]

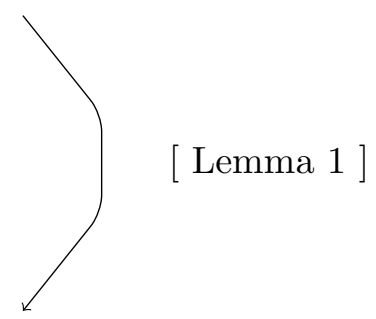

Figure 2: An illustration for the first case in the proof of Theorem 6, 
Then $G$ has an arc from $j$ to $V \backslash I$, hence, by definition, $Q$ has no $\operatorname{arc}$ from $j$ to $i$ and we deduce that $\tilde{G}$ has no arc from $j$ to $i$. Thus $\tilde{G}_{i} \cap B=\emptyset$ for every $i \in I$. We then deduce from Lemma 1, that there exists a degree-bounded system $\tilde{f}: \tilde{X} \rightarrow \tilde{X}$ on $\tilde{G}$ such that

(4) $\tilde{f}(\tilde{X}) \subseteq \tilde{h}(\tilde{Y})$,

(5) $\tilde{f}(x)=\tilde{h}(x)$ for all $x \in \tilde{Y}$ such that $x_{i}=\xi_{i}$ for all $i \in I$, that is, for all $x \in Y$,

(6) $\tilde{f}_{i}(x)=\tilde{h}_{i}(x)$ for all $x \in \tilde{Y}$ and $i \in I$.

As a consequence, $\tilde{f}$ has the following four properties:

(7) $\tilde{f}_{i}(\tilde{X}) \subseteq h_{i}(Y)$ for all $i \notin I$,

(8) $\tilde{f}_{i}^{\ell}(\tilde{X}) \subseteq \tilde{h}_{i}^{\ell}(\tilde{Y})$ for all $i \in I$ and $\ell \geq 1$,

(9) $\tilde{f}_{i}^{\lambda(Q)+\beta(Q)}(\tilde{X}) \subseteq h_{i}(Y)$ for all $i \in I$,

(10) $\tilde{f}(x)=h(x)$ for all $x \in Y$.

Indeed, property (7) results from (1) and (4), and property (10) results from (5) and (3). We now prove (8) by induction on $\ell$. The case $\ell=1$ is given by (4), and if $\ell \geq 2$ and $i \in I$ then

$$
\tilde{f}_{i}^{\ell}(\tilde{X})=\tilde{f}_{i}\left(\tilde{f}^{\ell-1}(\tilde{X})\right) \subseteq \tilde{f}_{i}\left(\tilde{h}^{\ell-1}(\tilde{Y})\right)=\tilde{h}_{i}^{\ell}(\tilde{Y}),
$$

where the inclusion results from the induction hypothesis and the fact that $\tilde{f}_{i}$ only depends on variables with indices in $I$ (because $\tilde{G}$ has no arc from $V \backslash I$ to $I$ ), and where the last equality results from (6). Finally, (9) results from (8) and (2).

Let $A$ be the set of sources of $\tilde{G}$ that are not sources of $G$, and let $B$ be the set of sinks of $\tilde{G}$ that are not sinks of $G$. Since $G$ is obtained from $\tilde{G}$ by adding only $\operatorname{arcs}(i, j, s)$ with $j \in I$ (see Figure (2), we have $A \subseteq I$. Let us prove that $B$ is empty. Let $i$ be a sink of $\tilde{G}$. If $i \notin I$ then $i$ is a sink of $H \backslash I$ and thus, by hypothesis, $i$ is a sink of $G$. If $i \in I$ then, by the definition of $\tilde{G}$, $G$ has no arc from $i$ to $V \backslash I$ thus $i$ is a sink of $G$, and since $i$ is a sink of $\tilde{H}$, by the definition of $\tilde{H}, G$ has no arc from $i$ to $I$. Thus $i$ is a sink of $G$. This proves that $B=\emptyset$. We deduce that $G$ has no arc from a sink of $\tilde{G}$ to a source of $\tilde{G}$. Now, suppose, for a contradiction, that $G$ has an arc from a vertex of $\tilde{G}$ to an isolated vertex $i$ of $\tilde{G}$. If $i \notin I$ then $i$ is isolated in $H \backslash I$ but not in $G$, a contradiction, thus $i \in I$. Hence, $i$ is a trivial connected component of $G[I]$, which is strongly connected, which has no leaving arcs, and which has at least one entering arc, and this contradicts the fact that $I$ has the property $P$. Thus $G$ has no $\operatorname{arc}$ from a vertex of $\tilde{G}$ to an isolated vertex of $\tilde{G}$. Thus $G$ and $\tilde{G}$ satisfy the conditions of Lemma 1, with $A \subseteq I$ and $B=\emptyset$. Therefore, there exists a degree-bounded system $f: X \rightarrow X$ on $G$ such that

(11) $f(X) \subseteq \tilde{X}$

(12) $f_{i}(X) \subseteq \tilde{f}_{i}(\tilde{X})$ for all $i \notin A$,

(13) $f(x)=\tilde{f}(x)$ for all $x \in \tilde{X}$.

As a consequence, $f$ has the following properties:

(14) $f_{i}(X) \subseteq h_{i}(Y)$ for all $i \notin I$,

(15) $f_{i}^{|I|+1}(X) \subseteq h_{i}(Y)$ for all $i \in I$,

(16) $f(x)=h(x)$ for all $x \in Y$. 
Indeed, (14) results from (12), (7) and the fact that $A \subseteq I$. Also, (16) results from (13) and (10). It remains to prove (15). Suppose first that $A=\emptyset$. Then following (12) we have $f(X) \subseteq \tilde{f}(\tilde{X})$ and we deduce from (13) that $f^{\ell}(X) \subseteq \tilde{f}^{\ell}(\tilde{X})$ for all $\ell \geq 1$. Thus by (9) we have

$$
f_{i}^{\lambda(Q)+\beta(Q)}(X) \subseteq h_{i}(Y) .
$$

Since $\lambda(Q) \leq|I|$ and $\beta(Q) \leq 1$ we obtain (15). Suppose now that $A \neq \emptyset$. Then following (11) and (13) we have $f^{\ell+1}(X) \subseteq \tilde{f}^{\ell}(\tilde{X})$ for all $\ell \geq 0$. Thus by (9) we have

$$
f_{i}^{\lambda(Q)+\beta(Q)+1}(X) \subseteq h_{i}(Y)
$$

If $Q$ is basic then $\beta(Q)=0$ and (15) follows. Otherwise, $Q$ is not basic, and since $A \neq \emptyset$ and $A \subseteq I, Q$ has a source. Hence, $Q$ has at least two distinct initial strong components. Thus, as explained in Remark 1, we have $\lambda(Q)<|I|$ and (15) follows.

We deduce from (14), (15) and (16) that $f$ converges toward $h$ in at most $|I|+1$ steps.

Case 2: $\boldsymbol{I}$ has not the property $\boldsymbol{P}$. Let $I_{1}$ be the set of vertices that belongs to a connected component $F$ of $G[I]$ such that $F$ is not strongly connected, or has a leaving arc, or has no entering arcs. Let $J=I \backslash \tilde{I}$. Since $I$ has not the property $P, J$ is not empty. Let $\tilde{G}$ be the signed digraph obtained from $G$ by removing every arc $(i, j, s)$ with $j \in J$ (see Figure 3). Then, $\tilde{I}$ is the set of vertices isolated in $H$ but not in $\tilde{G}$. Furthermore, it is straightforward to check that no connected component of $\tilde{G}$ is a cycle contained in $\tilde{G}[\tilde{I}]$ and that every source (resp. sink) of $\tilde{G} \backslash \tilde{I}$ is a source (resp. sink) of $\tilde{G}$. Furthermore, since $\tilde{I}$ has the property $P$ with respect to $\tilde{G}$, according to the first case, there exists a degree-bounded system $\tilde{f}: \tilde{X} \rightarrow \tilde{X}$ on $\tilde{G}$ such that

(1) $\tilde{f}_{i}(\tilde{X}) \subseteq h_{i}(Y)$ for all $i \notin \tilde{I}$,

(2) $\tilde{f}_{i}^{|\tilde{I}|+1}(\tilde{X}) \subseteq h_{i}(Y)$ for all $i \in \tilde{I}$, and thus $\tilde{f}_{i}^{|I|+1}(\tilde{X}) \subseteq h_{i}(Y)$ for all $i \in \tilde{I}$,

(3) $\tilde{f}(x)=h(x)$ for all $x \in Y$.

Let $\xi \in \mathbb{Z}^{n}$ be defined by $\xi_{i}=\max \left(\tilde{X}_{i}\right)$ for all $i \in\{1, \ldots, n\}$. Let $J=I \backslash \tilde{I}$ and let $Q$ be the spanning subgraph of $G$ that only contains the arcs $(i, j, s)$ of $G$ with $j \in J$. So $\tilde{G}$ and $Q$ are arc-disjoint and $G=\tilde{G} \cup Q$ (see Figure [3). Since every connected components $G[J]$ is strongly connected, has no leaving arcs and has an entering arc, we deduce that every connected component of $Q$ is basic. Hence, by applying Theorem 5 on each connected component of $H$ (cf. Remark 3) we deduce that there exists a degree-bounded system $g: Z \rightarrow Z$ on $Q$ such that

$$
g^{\lambda(Q)}=\operatorname{cst}=\xi .
$$

and such that $\xi_{i}=\min \left(Z_{i}\right)$ for all the sources $i$ of $Q$. Since if $i \notin J$ then $i$ is a source of $Q$ we have $\max \left(\tilde{X}_{i}\right)=\xi_{i}=\min \left(Z_{i}\right)$ for all $i \notin J$. Remark also that $Y_{i}=\tilde{X}_{i}=\left\{\xi_{i}\right\} \subseteq Z_{i}$ for all $i \in J$.

Let $X$ be defined by:

$$
X=X_{1} \times \cdots \times X_{n} \quad \text { with } \quad X_{i}= \begin{cases}\tilde{X}_{i} \cup Z_{i} & \text { if } i \notin J \\ Z_{i} & \text { if } i \in J .\end{cases}
$$

Let $\pi: X \rightarrow Z$ and $\tilde{\pi}: X \rightarrow \tilde{X}$ be the surjections defined by:

$$
\pi_{i}(x)=\left\{\begin{array}{ll}
\max \left(x_{i}, \xi_{i}\right) & \text { if } i \notin J \\
x_{i} & \text { if } i \in J .
\end{array} \text { and } \quad \tilde{\pi}_{i}(x)= \begin{cases}\min \left(x_{i}, \xi_{i}\right) & \text { if } i \notin J \\
\xi_{i} & \text { if } i \in J\end{cases}\right.
$$

Finally, let $f: X \rightarrow X$ be defined by:

$$
f_{i}(x)= \begin{cases}\tilde{f}_{i}(\tilde{\pi}(x)) & \text { if } i \notin J \\ g_{i}(\pi(x)) & \text { if } i \in J .\end{cases}
$$

Since $\tilde{f}$ is a degree-bounded system on $\tilde{G}$ and $g$ is a degree-bounded system on $Q$, it is easy to check that $f$ is a degree-bounded system on $G=\tilde{G} \cup Q$. $f$ has also the following properties: 


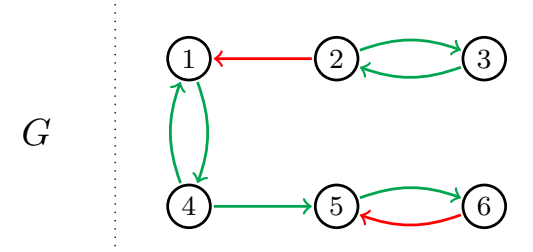

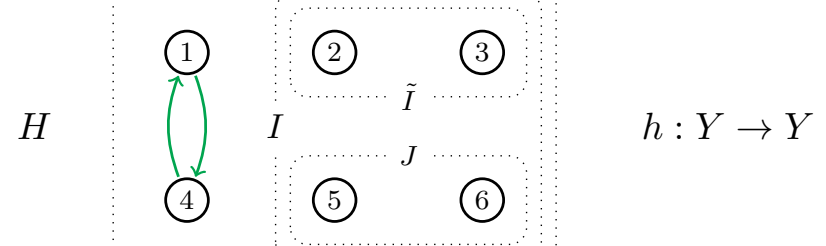
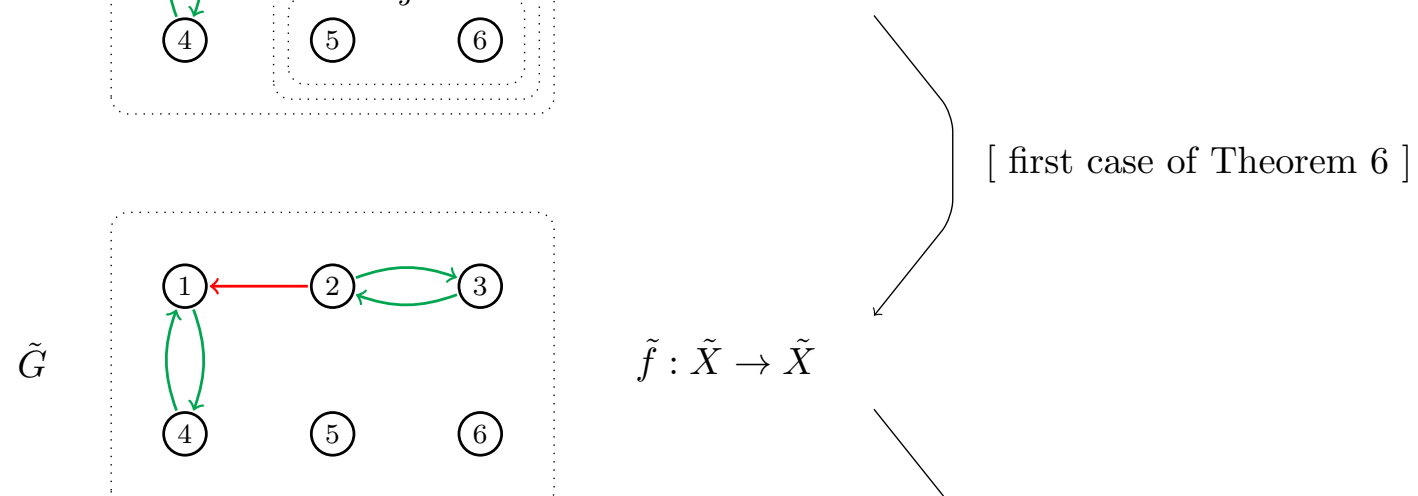

(1) (2) (3)

$Q$

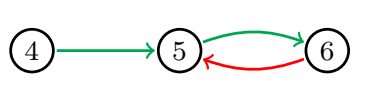

$g: Z \rightarrow Z$

[ Theorem [5]
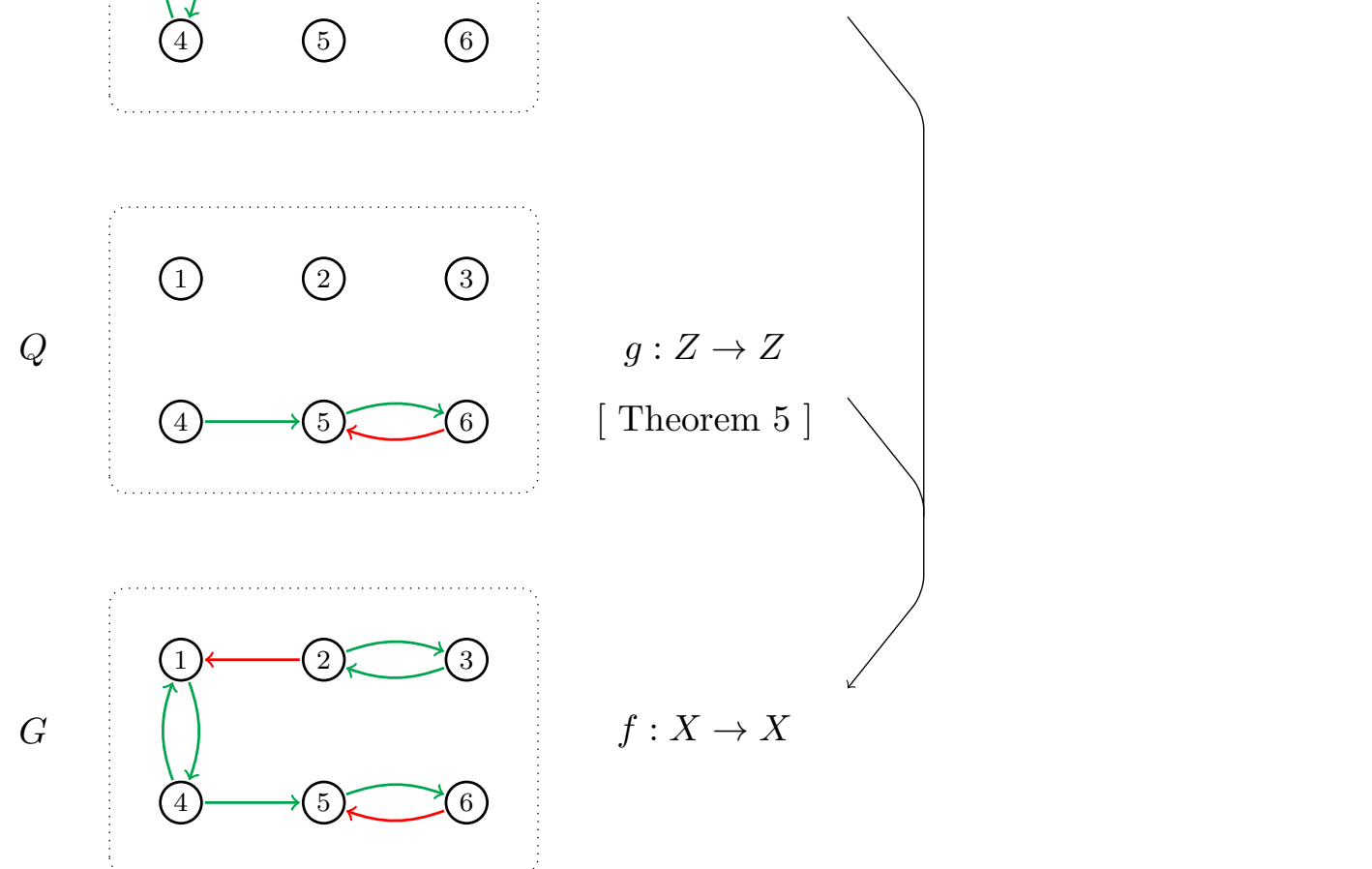

Figure 3: An illustration for the second case in the proof of Theorem 6. 
(4) $f_{i}^{\ell}(X) \subseteq \tilde{f}_{i}^{\ell}(\tilde{X})$ for all $i \notin J$ and $\ell \geq 1$.

(5) $f_{i}^{\ell}(x)=g_{i}^{\ell}(\pi(x))$ for all $i \in J, x \in X$ and $\ell \geq 1$, so that $f_{i}^{\lambda(Q)}=\operatorname{cst}=\xi_{i}$ for all $i \in J$,

(6) $f(x)=\tilde{f}(x)$ for all $x \in \tilde{X}$.

We first prove (5) by induction on $\ell$. The case $\ell=1$ results from the definition of $f_{i}$ for $i \in J$. If $\ell \geq 2$ then, by definition, we have

$$
f_{i}^{\ell}(x)=f_{i}\left(f^{\ell-1}(x)\right)=g_{i}\left(\pi\left(f^{\ell-1}(x)\right)\right) .
$$

By induction hypothesis, for all $j \in J$ we have $f_{j}^{\ell-1}(x)=g_{j}^{\ell-1}(\pi(x)) \geq \xi_{j}$ thus $\pi_{j}\left(f^{\ell-1}(x)\right)=$ $g_{j}^{\ell-1}(\pi(x))$. If $j \notin J$ then $f_{j}^{\ell-1}(x) \leq \xi_{j}$ thus $\pi_{j}\left(f^{\ell-1}(x)\right)=\xi_{j}$. Since $j$ is a source of $Q$ and $\xi$ is a fixed point of $g$, we have $g_{j}=$ cst $=\xi_{j}$. Thus, $\pi_{j}\left(f^{\ell-1}(x)\right)=g_{j}^{\ell-1}(\pi(x))$. Consequently,

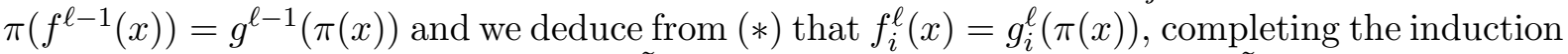
step. We now prove (6). Let $x \in \tilde{X}$. Then $\tilde{\pi}(x)=x$ thus $f_{i}(x)=\tilde{f}_{i}(x)$ for all $i \notin J$. Furthermore, $\pi(x)=\xi$ and if $i \in I$ then $\tilde{X}_{i}=\left\{\xi_{i}\right\}=\left\{x_{i}\right\}$. Since $\xi$ is a fixed point of $g$ we deduce that $f_{i}(x)=g_{i}(\xi)=\xi_{i}=\tilde{f}_{i}(x)$. Hence $f(x)=\tilde{f}(x)$ and (6) is proved. We finally prove (4) by induction on $\ell$. The case $\ell=1$ is an immediate consequence of the definition of $f_{i}$ for $i \notin J$. If $\ell \geq 2$ and $i \notin J$ then

$$
f_{i}^{\ell}(X)=f_{i}\left(\tilde{f}^{\ell-1}(\tilde{X})\right) \subseteq f_{i}\left(\tilde{f}^{\ell-1}(\tilde{X})\right)=\tilde{f}_{i}^{\ell}(\tilde{X}),
$$

where the inclusion results from the induction hypothesis and the fact that $f_{i}$ only depends on variables with indices which are not in $J$ (that is, $\tilde{G}$ has no arc from $J$ to $V \backslash J$ ), and where the last equality results from (6).

As a consequence, $f$ has the following three properties:

(7) $f_{i}(X) \subseteq h_{i}(Y)$ for all $i \notin I$.

(8) $f_{i}^{|I|+1}(X) \subseteq h_{i}(Y)$ for all $i \in I$.

(9) $f(x)=h(x)$ for all $x \in Y$.

Indeed, (7) results from (4) and (1), and (9) results from (6) and (3). It remains to prove (8). From (4) and (2) we have $f_{i}^{|I|+1}(X) \subseteq h_{i}(Y)$ for all $i \in \tilde{I}$, and from (5) we have $f_{i}^{\ell}(X)=\left\{\xi_{i}\right\}=$ $h_{i}(Y)$ for all $i \in J$ and $\ell \geq \lambda(Q)$. We deduce that is sufficient to prove that $\lambda(Q) \leq|I|+1$. Since $J$ corresponds to the set of non-sources of $Q$ and $Q$ is basic, by Remark 1, we have $\lambda(Q) \leq|J|+1 \leq|I|+1$. This completes the proof of (8).

We deduce from (7), (8) and (9) that $f$ converges toward $h$ in at most $|I|+1$ steps.

We now prove Lemma 1, using the following notations. For all $X \subseteq \mathbb{Z}^{n}$ and $y \in \mathbb{Z}^{n}$, $X+y=\{x+y \mid x \in X\}$. We denote by $e_{i}$ the $n$-tuple whose components are all equal to 0 , excepted th $i$ th one, which is equal to 1 .

Proof of Lemma 1. We proceed by induction on the number of $\operatorname{arcs}$ in $G$ that are not in $H$. If no such arcs exist, then $G=H$ and there is nothing to prove. So assume that $G$ has an arc

$$
a=(j, i, s)
$$

that is not in $H$. Let $\tilde{G}=G \backslash a$ be the spanning subgraph obtained from $G$ by removing $a$. Let $\tilde{A}$ be the set of sources of $H$ that are not sources of $\tilde{G}$ and let $\tilde{B}$ be the set of sinks of $H$ that are no sinks of $\tilde{G}$. By induction hypothesis, there exists a degree-bounded system $\tilde{f}: \tilde{X} \rightarrow \tilde{X}$ on $\tilde{G}$ such that

(i) $\tilde{f}(\tilde{X}) \subseteq Y$, 
(ii) $\tilde{f}_{k}(\tilde{X}) \subseteq h_{k}(Y)$ for all $k \notin \tilde{A}$.

(iii) $\tilde{f}(x)=h(x)$ for all $x \in Y$ such that $x_{k}=\xi_{k}$ for all $k \in \tilde{B}$.

(iv) $\tilde{f}_{k}(x)=h_{k}(x)$ for all $x \in Y$ and vertex $k$ such that $\tilde{G}_{k} \cap \tilde{B}=\emptyset$.

Since $G$ has no arc from a source of $H$ to a sink of $H, G$ has no arc from a source of $\tilde{G}$ to a sink of $\tilde{G}$. Furthermore, since $G$ has no arc from a vertex of $H$ to an isolated vertex of $H, G$ has no arc from a vertex of $\tilde{G}$ to an isolated vertex of $\tilde{G}$. This leaves the following three possibilities.

Case 1: $j$ is not a sink of $\tilde{G}$ and $i$ is not a source of $\tilde{G}$.

- If $s=+$ we set $X=\tilde{X} \cup\left(\tilde{X}+e_{j}\right)$ and define $f: X \rightarrow X$ as follows:

$$
\begin{aligned}
& f_{i}(x)= \begin{cases}\max \left(\tilde{f}_{i}(\tilde{X})\right) & \text { if } x_{j}=\max \left(X_{j}\right) \\
\tilde{f}_{i}(x) & \text { otherwise }\end{cases} \\
& f_{k}(x)=\left\{\begin{array}{ll}
\tilde{f}_{k}\left(x-e_{j}\right) & \text { if } x \notin \tilde{X} \\
\tilde{f}_{k}(x) & \text { otherwise }
\end{array} \quad \forall k \neq i .\right.
\end{aligned}
$$

Hence, $f(X) \subseteq \tilde{f}(\tilde{X})$ and $f(x)=\tilde{f}(x)$ for all $x \in \tilde{X}$. Since $i$ is a not a sink of $\tilde{G}$ we have $A=\tilde{A}$ and since $j$ is not a source of $\tilde{G}$, we have $B=\tilde{B}$. Thus, using the properties (i)-(iv), we deduce that $f$ satisfies the four points of the statement. It remains to prove that $f$ is a degree-bounded system on $G$.

Let $G_{f}$ be the interaction graph of $f$. Since $f(x)=\tilde{f}(x)$ for all $x \in \tilde{X}, \tilde{G}$ is a subgraph of $G_{f}$. Since it is straightforward to prove that $G_{f}$ is a subgraph of $G$, to prove that $G_{f^{\prime}}=G$, it is sufficient to prove that $a$ is an $\operatorname{arc}$ of $G_{f}$. Let $x \in X$ be such that $f_{i}(x)=\min \left(\tilde{f}_{i}(\tilde{X})\right)$ and suppose that $x_{j}$ is maximal for this property. Since $i$ is not a source of $\tilde{G}$, we have $\min \left(\tilde{f}_{i}(\tilde{X})\right)<\max \left(\tilde{f}_{i}(\tilde{X})\right)$ and we deduce from the definition of $f_{i}$ that $x_{j}<\max \left(X_{j}\right)$. Consequently, $f_{i}\left(x+e_{j}\right)>\min \left(\tilde{f}_{i}(\tilde{X})\right)$ so $G_{f}$ has a positive $\operatorname{arc}$ from $j$ to $i$. Thus $G_{f}=G$, that is, $f$ is an FDS on $G$. Since $\tilde{f}$ is degree-bounded, it is straightforward to prove that $f$ is degree-bounded too.

- If $s=-$, we proceed similarly with $X=\tilde{X} \cup\left(\tilde{X}+e_{j}\right)$ and $f: X \rightarrow X$ defined by:

$$
\begin{aligned}
& f_{i}(x)= \begin{cases}\min \left(\tilde{f}_{i}(\tilde{X})\right) & \text { if } x_{j}=\max \left(X_{j}\right) \\
\tilde{f}_{i}(x) & \text { otherwise }\end{cases} \\
& f_{k}(x)=\left\{\begin{array}{ll}
\tilde{f}_{k}\left(x-e_{j}\right) & \text { if } x \notin \tilde{X} \\
\tilde{f}_{k}(x) & \text { otherwise }
\end{array} \quad \forall k \neq i .\right.
\end{aligned}
$$

Case 2: $\boldsymbol{j}$ is a sink of $\tilde{G}$ and $i$ is not a source of $\tilde{G}$. Since $j$ is a sink of $\tilde{G}$ we have $\left|\tilde{X}_{j}\right|=1$ if $j$ is isolated in $\tilde{G}$, and $\left|\tilde{X}_{j}\right|=2$ otherwise. Let

$$
X= \begin{cases}\tilde{X} & \text { if }\left|\tilde{X}_{j}\right|=2 \\ \tilde{X} \cup\left(\tilde{X}+e_{j}\right) & \text { if }\left|\tilde{X}_{j}\right|=1\end{cases}
$$

Hence $\left|X_{j}\right|=2$, so either $\xi_{j}<\max \left(X_{j}\right)$ or $\xi_{j}>\min \left(X_{j}\right)$, and we consider four cases.

- If $s=+$ and $\xi_{j}<\max \left(X_{j}\right)$, we define $f: X \rightarrow X$ by:

$$
\begin{aligned}
& f_{i}(x)= \begin{cases}\max \left(\tilde{f}_{i}(\tilde{X})\right) & \text { if } x_{j}=\max \left(X_{j}\right) \\
\tilde{f}_{i}(x) & \text { otherwise }\end{cases} \\
& f_{k}(x)=\left\{\begin{array}{ll}
\tilde{f}_{k}\left(x-e_{j}\right) & \text { if } x \notin \tilde{X} \\
\tilde{f}_{k}(x) & \text { otherwise }
\end{array} \quad \forall k \neq i .\right.
\end{aligned}
$$


Hence, $f(X) \subseteq \tilde{f}(\tilde{X})$ and $f(x)=\tilde{f}(x)$ for all $x \in X$ with $x_{j}=\xi_{j}$. Since $i$ is a not a source of $\tilde{G}$ we have $A=\tilde{A}$, we deduce from (i) and (ii) that $f$ satisfies the first two points of the statement. Since $B=\tilde{B} \cup\{j\}$ and $j \in G_{i} \cap B$, we then deduce from (iii) and (iv) that $f$ satisfies the last two points. It remains to prove that $f$ is a degree-bounded system on $G$. We prove that $G$ is the interaction graph of $f$ exactly as in the previous case. Next, since $\tilde{f}$ is a degree-bounded system on $\tilde{G}$, and since $\left|X_{j}\right|=2=d_{G}^{\text {out }}(j)+1$, we deduce that $f$ is degree-bounded. Thus $f$ is indeed a degree-bounded system on $G$.

- If $s=+$ and $\xi_{j}>\min \left(X_{j}\right)$, we proceed similarly with $f: X \rightarrow X$ defined by:

$$
\begin{aligned}
& f_{i}(x)= \begin{cases}\min \left(\tilde{f}_{i}(\tilde{X})\right) & \text { if } x_{j}=\min \left(X_{j}\right) \\
\tilde{f}_{i}(x) & \text { otherwise }\end{cases} \\
& f_{k}(x)=\left\{\begin{array}{ll}
\tilde{f}_{k}\left(x-e_{j}\right) & \text { if } x \notin \tilde{X} \\
\tilde{f}_{k}(x) & \text { otherwise }
\end{array} \quad \forall k \neq i .\right.
\end{aligned}
$$

- If $s=-$ and $\xi_{j}<\max \left(X_{j}\right)$, we proceed similarly with $f: X \rightarrow X$ defined by:

$$
\begin{aligned}
& f_{i}(x)= \begin{cases}\min \left(\tilde{f}_{i}(\tilde{X})\right) & \text { if } x_{j}=\max \left(X_{j}\right) \\
\tilde{f}_{i}(x) & \text { otherwise }\end{cases} \\
& f_{k}(x)=\left\{\begin{array}{ll}
\tilde{f}_{k}\left(x-e_{j}\right) & \text { if } x \notin \tilde{X} \\
\tilde{f}_{k}(x) & \text { otherwise }
\end{array} \quad \forall k \neq i .\right.
\end{aligned}
$$

- If $s=-$ and $\xi_{j}>\min \left(X_{j}\right)$, we proceed similarly with $f: X \rightarrow X$ defined by:

$$
\begin{aligned}
& f_{i}(x)= \begin{cases}\max \left(\tilde{f}_{i}(\tilde{X})\right) & \text { if } x_{j}=\min \left(X_{j}\right) \\
\tilde{f}_{i}(x) & \text { otherwise }\end{cases} \\
& f_{k}(x)=\left\{\begin{array}{ll}
\tilde{f}_{k}\left(x-e_{j}\right) & \text { if } x \notin \tilde{X} \\
\tilde{f}_{k}(x) & \text { otherwise }
\end{array} \quad \forall k \neq i .\right.
\end{aligned}
$$

Case 3: $\boldsymbol{j}$ is not a sink of $\tilde{G}$ and $i$ is a non-isolated source of $\tilde{G}$. Then $i$ is a source of both $\tilde{G}$ and $H$, thus $\tilde{f}_{i}=\operatorname{cst}=c_{i}$ and $h_{i}=\operatorname{cst}=c_{i}^{\prime}$, and following (iii) $c_{i}=c_{i}^{\prime}$. Since $i$ is not isolated in $\tilde{G}$ we have $\left|\tilde{X}_{i}\right| \geq 2$ and thus either $c_{i}<\max \left(\tilde{X}_{i}\right)$ or $c_{i}>\min \left(\tilde{X}_{i}\right)$, and we consider the following four cases. In every cases we set

$$
X=\tilde{X} \cup\left(\tilde{X}+e_{j}\right) .
$$

- If $s=+$ and $c_{i}<\max \left(\tilde{X}_{i}\right)$, we define $f: X \rightarrow X$ by:

$$
\begin{aligned}
& f_{i}(x)= \begin{cases}\max \left(\tilde{X}_{i}\right) & \text { if } x_{j}=\max \left(X_{j}\right) \\
c_{i} & \text { otherwise }\end{cases} \\
& f_{k}(x)=\left\{\begin{array}{ll}
\tilde{f}_{k}\left(x-e_{j}\right) & \text { if } x \notin \tilde{X} \\
\tilde{f}_{k}(x) & \text { otherwise }
\end{array} \quad \forall k \neq i .\right.
\end{aligned}
$$

Hence, $f_{i}(X) \subseteq \tilde{X}_{i}$ and $f_{k}(X) \subseteq \tilde{f}_{k}(\tilde{X})$ for all $k \neq i$. Since $A=\tilde{A} \cup\{i\}$ we deduce from (i) and (ii) that $f$ satisfies the first two points of the statement. Since $B=\tilde{B}$ and $h(x)=\tilde{f}(x)$ for all $x \in \tilde{X}$, we deduce from (iii) and (iv) that $f$ satisfies the last two points of the statement. It remains to prove that $f$ is a degree-bounded system on $G$.

Let $G_{f}$ be the interaction graph of $f$. Since $f(x)=\tilde{f}(x)$ for all $x \in \tilde{X}, \tilde{G}$ is a subgraph of $G_{f}$. Since it is straightforward to show that $G_{f}$ is a subgraph of $G$, to prove that $G_{f}=G$, it is sufficient to prove that $a$ is an arc of $G_{f}$. Let $x \in X$ with $x_{j}=\max \left(X_{j}\right)-1$. Then $f_{i}(x)=c_{i}<\max \left(\tilde{X}_{i}\right)$ and $f_{i}\left(x+e_{j}\right)=\max \left(\tilde{X}_{i}\right)$ thus $a$ is indeed an arc of $G_{f}$. Thus $f$ is a FDS on $G$, and since $\tilde{f}$ is degree-bounded, it is straightforward to prove that $f$ is degree-bounded too. 
- If $s=+$ and $c_{i}>\min \left(\tilde{X}_{i}\right)$ we proceed similarly with $f: X \rightarrow X$ define by:

$$
\begin{aligned}
f_{i}(x) & = \begin{cases}c_{i} & \text { if } x_{j}=\max \left(X_{j}\right) \\
\min \left(\tilde{X}_{i}\right) & \text { otherwise }\end{cases} \\
f_{k}(x) & =\left\{\begin{array}{ll}
\tilde{f}_{k}\left(x-e_{j}\right) & \text { if } x \notin \tilde{X} \\
\tilde{f}_{k}(x) & \text { otherwise }
\end{array} \quad \forall k \neq i .\right.
\end{aligned}
$$

- If $s=-$ and $c_{i}<\max \left(\tilde{X}_{i}\right)$, we proceed similarly with $f: X \rightarrow X$ defined by:

$$
\begin{aligned}
f_{i}(x) & = \begin{cases}c_{i} & \text { if } x_{j}=\max \left(X_{j}\right) \\
\max \left(\tilde{X}_{i}\right) & \text { otherwise }\end{cases} \\
f_{k}(x) & =\left\{\begin{array}{ll}
\tilde{f}_{k}\left(x-e_{j}\right) & \text { if } x \notin \tilde{X} \\
\tilde{f}_{k}(x) & \text { otherwise }
\end{array} \quad \forall k \neq i .\right.
\end{aligned}
$$

- If $s=-$ and $c_{i}>\min \left(X_{i}\right)$, we proceed similarly with $f: X \rightarrow X$ defined by:

$$
\begin{aligned}
f_{i}(x) & = \begin{cases}\min \left(\tilde{X}_{i}\right) & \text { if } x_{j}=\max \left(X_{j}\right) \\
c_{i} & \text { otherwise }\end{cases} \\
f_{k}(x) & =\left\{\begin{array}{ll}
\tilde{f}_{k}\left(x-e_{j}\right) & \text { if } x \notin \tilde{X} \\
\tilde{f}_{k}(x) & \text { otherwise }
\end{array} \quad \forall k \neq i .\right.
\end{aligned}
$$

Acknowledgment I wish to thank Emmanuel Bonan and Tony Silva for stimulating discussions.

\section{References}

[1] W. Abou-Jaoudé, P. Monteiro, A. Naldi, M. Grandclaudon, V. Soumelis, C. Chaouiya, and D. Thieffry. Model checking to assess t-helper cell plasticity. Frontiers in bioengineering and biotechnology, 2, 2014.

[2] J. Aracena. Maximum number of fixed points in regulatory Boolean networks. Bulletin of Mathematical Biology, 70(5):1398-1409, 2008.

[3] J. Aracena, A. Richard, and L. Salinas. Number of fixed points and disjoint cycles in monotone boolean networks. SIAM Journal on Discrete Mathematics, 31(3):1702-1725, 2017.

[4] O. Cinquin and J. Demongeot. Positive and negative feedback: strinking a balance between necessary antagonists. Journal of Theoretical Biology, 216:229-241, 2002.

[5] M. Gadouleau, A. Richard, and E. Fanchon. Reduction and fixed points of boolean networks and linear network coding solvability. IEEE Transactions on Information Theory, 62(5):2504-2519, 2016.

[6] M. Gadouleau and S. Riis. Graph-theoretical constructions for graph entropy and network coding based communications. IEEE Transactions on Information Theory, 57(10):6703$6717,2011$.

[7] Maximilien Gadouleau and Adrien Richard. Simple dynamics on graphs. Theoretical Computer Science, 628:62-77, 2016. 
[8] E. Goles and S. Martínez. Neural and Automata Networks: Dynamical Behavior and Applications. Kluwer Academic Publishers, 1990.

[9] J.L. Gouzé. Positive and negative circuits in dynamical systems. Journal of Biological Systems, 6:11-15, 1998.

[10] J. Hopfield. Neural networks and physical systems with emergent collective computational abilities. Proc. Nat. Acad. Sc. U.S.A., 79:2554-2558, 1982.

[11] S. A. Kauffman. Metabolic stability and epigenesis in randomly connected nets. Journal of Theoretical Biology, 22:437-467, 1969.

[12] S. A. Kauffman. Origins of Order Self-Organization and Selection in Evolution. Oxford University Press, 1993.

[13] M. Kaufman and C. Soulé. On the multistationarity of chemical reaction networks. Journal of theoretical biology, 465:126-133, 2019.

[14] M. Kaufman, C. Soulé, and R. Thomas. A new necessary condition on interaction graphs for multistationarity. Journal of Theoretical Biology, 248(4):675-685, 2007.

[15] N. Le Novère. Quantitative and logic modelling of molecular and gene networks. Nature Reviews Genetics, 16:146-158, 2015.

[16] W. S. Mac Culloch and W. S. Pitts. A logical calculus of the ideas immanent in nervous activity. Bull. Math Bio. Phys., 5:113-115, 1943.

[17] E. Plathe, T. Mestl, and S.W. Omholt. Feedback loops, stability and multistationarity in dynamical systems. Journal of Biological Systems, 3:569-577, 1995.

[18] E. Remy, P. Ruet, and D. Thieffry. Graphic requirements for multistability and attractive cycles in a Boolean dynamical framework. Advances in Applied Mathematics, 41(3):335-350, 2008.

[19] A. Richard. An extension of a combinatorial fixed point theorem of Shih and Dong. Advances in Applied Mathematics, 41(4):620-627, 2008.

[20] A. Richard. Positive circuits and maximal number of fixed points in discrete dynamical systems. Discrete Applied Mathematics, 157(15):3281-3288, 2009.

[21] A. Richard. Negative circuits and sustained oscillations in asynchronous automata networks. Advances in Applied Mathematics, 44(4):378-392, 2010.

[22] A. Richard. Fixed points and connections between positive and negative cycles in boolean networks. Discrete Applied Mathematics, 2018.

[23] A. Richard. Positive and negative cycles in boolean networks. Journal of theoretical biology, 463:67-76, 2019.

[24] A. Richard and J.-P. Comet. Necessary conditions for multistationarity in discrete dynamical systems. Discrete Applied Mathematics, 155(18):2403-2413, 2007.

[25] F. Robert. Discrete iterations: a metric study, volume 6 of Series in Computational Mathematics. Springer, 1986.

[26] F. Robert. Les systèmes dynamiques discrets, volume 19 of Mathématiques et Applications. Springer, 1995. 
[27] E.H. Snoussi. Necessary conditions for multistationarity and stable periodicity. Journal of Biological Systems, 6:3-9, 1998.

[28] S. Soliman. A stronger necessary condition for the multistationarity of chemical reaction networks. Bulletin of mathematical biology, 75(11):2289-2303, 2013.

[29] C. Soulé. Graphical requirements for multistationarity. ComPlexUs, 1:123-133, 2003.

[30] C. Soulé. Mathematical approaches to differentiation and gene regulation. C.R. Paris Biologies, 329:13-20, 2006.

[31] R. Thomas. Boolean formalization of genetic control circuits. Journal of Theoretical Biology, 42(3):563-585, 1973.

[32] R. Thomas. On the relation between the logical structure of systems and their ability to generate multiple steady states or sustained oscillations. Springer Series in Synergies 9, pages 180-193, 1981.

[33] R. Thomas and R. d'Ari. Biological Feedback. CRC Press, 1990.

[34] R. Thomas and M. Kaufman. Multistationarity, the basis of cell differentiation and memory. I. structural conditions of multistationarity and other nontrivial behavior. Chaos: An Interdisciplinary Journal of Nonlinear Science, 11(1):170-179, 2001.

[35] R. Thomas and M. Kaufman. Multistationarity, the basis of cell differentiation and memory. II. Logical analysis of regulatory networks in terms of feedback circuits. Chaos: An Interdisciplinary Journal of Nonlinear Science, 11(1):180-195, 2001. 